\title{
Betacarotenemia con afectación nasal aislada en una niña: A propósito de un caso
} Betacarotenemia with isolated nose involvement in a child: a case report

\author{
Prof. Asoc. Dra. Burcu Kayhan-Tetika y Dra. Nilgün Çaylan ${ }^{b}$
}

\begin{abstract}
RESUMEN
La carotenemia es, en general, benigna y suele ser causada por la ingesta excesiva de alimentos ricos en betacaroteno. Su presentación clínica puede ocurrir a cualquier edad, pero es frecuente en los niños. En este artículo, describimos un caso raro de carotenemia con afectación nasal aislada con el objetivo de recordar la presencia de carotenemia en el diagnóstico diferencial de ictericia. A menudo, la carotenemia se reconoce fácilmente y la manejan los médicos de familia y los pediatras, que podrían participar potencialmente en el tratamiento de estos casos. También es importante conocer y reconocer la carotenemia para informar a las familias y evitar la realización de pruebas innecesarias.
\end{abstract}

Palabras clave: carotenemia, betacaroteno, ictericia, vitamina A.

http: / / dx.doi.org/10.5546/ aap.2019.e502

Texto completo en inglés:

http: / / dx.doi.org/10.5546/ aap.2019.eng.e502

Cómo citar: Kayhan-Tetik B, Çaylan N. Betacarotenemia con afectación nasal aislada en una niña: A propósito de un caso. Arch Argent Pediatr 2019;117(5):e502-e504.

\section{INTRODUCCIÓN}

La carotenemia es una entidad clínica caracterizada por la coloración amarillenta de la piel, resultado del aumento de la concentración sérica de betacaroteno y el depósito de caroteno principalmente en la capa córnea. Si bien la betacarotenemia se considera una enfermedad rara, se desconoce su prevalencia. Sin embargo,

a. Departamento de Medicina Familiar, Facultad de Medicina de la İnönü Üniversitesi, Malatya.

b. Instituto de Salud Pública, Departamento de Salud de Niños y Adolescentes, Ankara, Turquía.

\section{Correspondencia:}

Dra. Burch Kayhan-Tetik: drburcukayhan@hotmail.com

Financiamiento: Ninguno.

Conflicto de intereses: Ninguno que declarar.

Recibido: 6-8-2018

Aceptado: 25-2-2019 en un estudio de Priyadarshani realizado en 615 niños, se observó una prevalencia del $2 \% .{ }^{1}$ Esta presentación clínica puede ocurrir a cualquier edad, pero es frecuente en los niños. ${ }^{2}$ La carotenemia es, en general, benigna y suele ser causada por la ingesta excesiva de alimentos ricos en betacaroteno. Sin embargo, raramente está asociada con unos pocos trastornos metabólicos graves y enzimopatías congénitas que metabolizan los carotenoides. ${ }^{3}$

Presentamos este caso porque es rara la presencia de carotenemia aislada en la punta de la nariz y porque debe considerarse en el diagnóstico diferencial de la ictericia.

\section{CASO}

Se presentó una niña de 3 años con coloración amarillenta-anaranjada en la nariz de un mes de evolución y que, por lo demás, era sana. Según sus antecedentes clínicos, este color se observó por primera vez desde los 3 años y se repitió en cinco ocasiones. No presentaba síntomas generales y el color de su orina y sus heces era normal. Ningún miembro de su familia tenía antecedentes de síntomas similares. En el examen físico, se observó coloración amarillentaanaranjada en las aletas nasales y la punta de la nariz (Figura 1). El color de las palmas, las plantas de los pies, otras partes de la piel y las escleróticas era normal. El resto del examen físico estaba dentro de los límites habituales y su desarrollo y crecimiento eran normales. En los análisis de laboratorio, se observó que el hemograma, las pruebas funcionales renales y tiroideas y el perfil lipídico eran normales. Las pruebas funcionales hepáticas, la concentración sérica de bilirrubina y las pruebas serológicas de hepatitis A y $B$ estuvieron dentro de los límites habituales. La concentración sérica de betacaroteno era marcadamente elevada: $368 \mu \mathrm{mol} / 1$ (intervalo de referencia: 60-200 $\mu \mathrm{mol} / 1)$. La concentración sérica de vitamina A era normal: $72 \mu \mathrm{mol} / 1$ (intervalo de referencia: $30-80 \mu \mathrm{mol} / \mathrm{l}$ ). Al indagar sobre los detalles de la paciente, descubrimos que últimamente había consumido zanahoria, naranja y mandarina en exceso y que el color amarillento 
en la piel se había manifestado luego de esto. Se le diagnosticó carotenemia y se les recomendó a los padres que eliminaran las zanahorias, naranjas y mandarinas de la dieta porque son alimentos ricos en betacaroteno. En el seguimiento se observó que la paciente cumplió con la dieta y el color amarillento en la nariz desapareció por completo (Figura 2).

\section{DISCUSIÓN}

Los carotenoides son compuestos orgánicos que se hallan en muchas frutas y verduras de color rojo, naranja o amarillo y en las verduras de hojas verdes. Los alimentos muy ricos en carotenoides incluyen zanahoria, damasco, naranja, mandarina, mango, batata, col rizada, melón, papaya y otras verduras de hojas verdes. ${ }^{2}$ Entre los carotenoides, el betacaroteno es el más importante y se absorbe en el intestino delgado. Tras la absorción, se convierte en dos moléculas de retinol, un metabolito de la vitamina A en las células mucosas del intestino delgado, mediante la enzima betacaroteno $15,15^{\prime}$-dioxigenasa y luego ingresa al sistema linfático. ${ }^{4}$ Aproximadamente el $10 \%$ del betacaroteno ingerido ingresa a la circulación portal inalterado. Las lipoproteínas transportan el betacaroteno en el suero, que se elimina a través del colon y a través de la epidermis mediante el sudor y el material sebáceo. ${ }^{5}$

Los carotenoides comienzan a acumularse en la epidermis aproximadamente dos semanas después de lograr el equilibrio sérico. ${ }^{6}$ En general, la pigmentación amarilla del betacaroteno aparece cuando la concentración sérica supera los $250 \mathrm{mg} / \mathrm{dl} .^{2}$ La capa córnea es muy rica en lípidos, que tienen una afinidad por el caroteno; de este modo, el pigmento de caroteno se concentra en esta. ${ }^{2}$ La coloración amarillenta de la piel que

FIGURA 1. Coloración amarillenta en las aletas nasales y la punta de la nariz

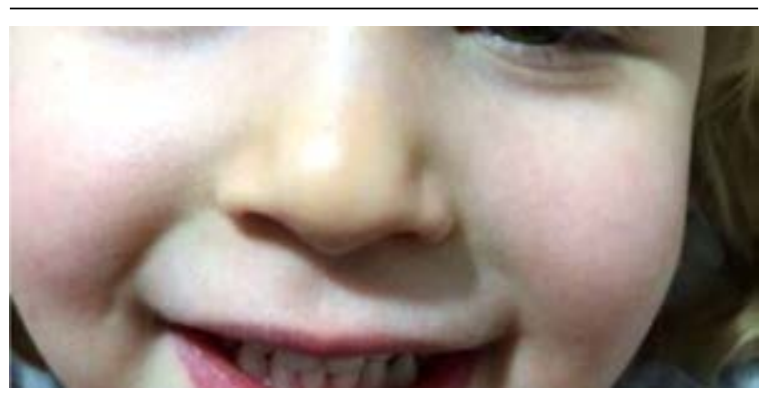

caracteriza la carotenemia suele manifestarse en áreas engrosadas de la capa córnea y en áreas con abundancia de glándulas sudoríparas. La pigmentación suele aparecer en las palmas y las plantas de los pies, los pliegues nasolabiales, la punta de la nariz, la pera, los nudillos y detrás de las orejas $y$, a veces, puede manifestarse en el paladar. ${ }^{2,5}$ Típicamente, no se ha observado afectación de las escleróticas en los casos de carotenemia. En nuestra paciente, solo se observó afectación de las aletas nasales y la punta de la nariz. No se observó afectación en otras áreas, en especial, las palmas y las plantas de los pies; las escleróticas estaban normales.

Al considerar el diagnóstico de carotenemia, el primer paso es excluir la ictericia. Si bien la ictericia escleral es un signo clínico temprano de la ictericia, los carotenoides no se acumulan en la esclerótica; la presencia de una esclerótica clara es un hallazgo clínico importante en el diagnóstico de la carotenemia. ${ }^{6}$ Además, en presencia de una enfermedad que produce ictericia, el cambio de color de la piel suele estar acompañado por otras manifestaciones, como fiebre, prurito, debilidad, vómitos, cambio de color de las heces y agrandamiento del hígado. Asimismo, el síndrome de Gilbert, un trastorno metabólico congénito, también es una de las enfermedades que puede confundirse con la carotenemia. En el diagnóstico diferencial, particularmente el síndrome de Gilbert se diagnostica por aumento de la bilirrubina indirecta en la sangre, junto con fatiga y estrés. El diagnóstico diferencial puede hacerse por la ausencia de aumento de la bilirrubina indirecta en la sangre. Una vez que se descartan la ictericia y el síndrome de Gilbert, se debe averiguar especialmente si existe un consumo excesivo de alimentos ricos en betacaroteno. En el caso de nuestra

FIGURA 2. La coloración amarillenta desapareció después de la restricción alimentaria

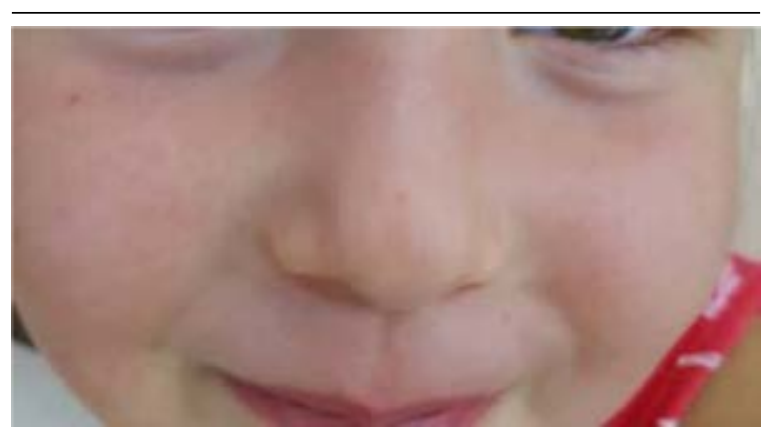


paciente, se mencionó el consumo excesivo de frutas que contienen caroteno. También se informaron casos de carotenemia con el consumo de suplementos nutritivos y alimentos procesados, así como de alimentos naturales que contienen carotenoides. ${ }^{6,7} \mathrm{Si}$ no se observa que la dieta sea causa de la carotenemia, el médico debe considerar otras afecciones, como diabetes mellitus, hipotiroidismo, anorexia nerviosa, hepatopatía y nefropatía. ${ }^{3}$

En general, el diagnóstico diferencial puede establecerse fácilmente con una buena historia clínica, un examen físico y análisis de laboratorio sencillos. Raramente, la carotenemia está asociada con una enzimopatía congénita que resulta de la deficiencia parcial o completa de 15,15'-dioxigenasa. Esta enzimopatía puede ser congénita y produce la acumulación de betacaroteno y una concentración de baja a normal de vitamina A..$^{8,9}$ En nuestra paciente, se creyó que la betacarotenemia dependía en gran medida de la dieta más que de una transición hereditaria porque la concentración de vitamina A era normal, no se hallaron manifestaciones clínicas previas similares en ningún familiar, se habían consumido en exceso alimentos ricos en betacaroteno y los síntomas respondieron a la restricción dietaria. Asimismo, es posible excluir fácilmente las enfermedades que pueden causar ictericia mediante la historia clínica, el examen físico y los análisis de laboratorio.

La piel amarillenta también puede ser por licopenemia, riboflavinemia, absorción percutánea o ingesta de ciertos medicamentos y químicos, como clorhidrato de quinacrina, azafrán, 2,4-dinitrofenol y ácido pícrico, y se los debe considerar en el diagnóstico diferencial de la carotenemia. ${ }^{3,9}$

\section{CONCLUSIÓN}

La carotenemia es, en general, una afección benigna y la ingesta excesiva de carotenoides no está asociada con toxicidad por vitamina A. A menudo, la carotenemia se reconoce fácilmente y la manejan los médicos de familia y los pediatras, que podrían participar potencialmente en el tratamiento de estos casos. También es importante conocer y reconocer la carotenemia para informar a las familias y evitar la realización de pruebas innecesarias.

\section{REFERENCIAS}

1. Priyadarshani AMB. Insights of hypercarotenaemia: A brief review. Clin Nutr ESPEN. 2018; 23:19-24.

2. Maharshak N, Shapiro J, Trau H. Carotenoderma-a review of the current literature. Int J Dermatol. 2003; 42(3):178-81.

3. Lascari AD. Carotenemia: A review. Clin Pediatr (Phila). 1981; 20(1):25-9.

4. Lakshman MR, Okoh C. Enzymatic conversion of all-transbeta-carotene to retinal. Methods Enzymol. 1993; 214:256-69.

5. Prince MR, Frisoli JK. Beta-carotene accumulation in serum and skin. Am J Clin Nutr. 1993; 57(2):175-81.

6. Karthik SV, Campbell-Davidson D, Isherwood D. Carotenemia in infancy and its association with prevalent feeding practices. Pediatr Dermatol. 2006; 23(6):571-3.

7. Takita Y, Ichimiya M, Hamamoto Y, Muto M. A case of carotenemia associated with ingestion of nutrient supplements. J Dermatol. 2006; 33(2):132-4.

8. Chattopadhyay M, Pramanik R, McGrath JA, Burrows NP. Familial carotenaemia and carotenoderma. Clin Exp Dermatol. 2014; 39(6):771-2.

9. Chattopadhyay M, Pramanik R, McGrath JA, Burrows NP. Familial carotenaemia and carotenoderma. Clin Exp Dermatol. 2014; 39(6):771-2. 